\title{
Quality of life, burden and satisfaction with care in caregivers of patients with a spinal cord injury during and after rehabilitation
}

\author{
Annemiek Petronella Maria Backx $\mathbb{D}^{1} \cdot$ Annemie Irene Frans Spooren ${ }^{2,3} \cdot$ Helena Maria Henrika Bongers-Janssen ${ }^{1,4}$. \\ Hanneke Bouwsema ${ }^{5,6}$
}

Received: 4 July 2017 / Revised: 13 March 2018 / Accepted: 14 March 2018 / Published online: 27 April 2018

(c) International Spinal Cord Society 2018

\begin{abstract}
Study design Longitudinal, prospective cohort study.

Objectives To examine the course that burden, quality of life $(\mathrm{QoL})$ and satisfaction with care taken in Dutch caregivers of patients with a SCI.

Setting Adelante Rehabilitation Centre and Dutch community, the Netherlands

Methods Caregiver Strain index (CSI), Short Form36 (SF-36) and Caregivers' Satisfaction with (Stroke) Care Questionnaire (C-SASC) were administered to caregivers $(n=37)$ of patients with a recently acquired SCI at the start of rehabilitation (T1), discharge from rehabilitation (T2) and at 8 weeks (T3), 6 months (T4) and 18 months after discharge (T5).

Results During rehabilitation, 20 caregivers (54\%) experienced high levels of burden (cutoff $>6$ ). CSI scores significantly decreased during follow-up (median CSI score T1:7 IQR[5,10], T5:4 IQR[1,7], $p=0.010$ ), at T5 5 caregivers (24\%) scored $>6$ on burden. Initial low scores on QoL improved significantly in the SF-36 domains 'social-functioning', 'emotional-rolefunctioning', 'mental health' and 'vitality'. Overall satisfaction with care of caregivers was good (C-SASC: median 3, IQR $[3,4])$ and stable over time. Moderate strongly negative correlations were found between total CSI-score and 'socialfunctioning' (T2-T3-T4), 'emotional-role-functioning' (all time points), 'mental health' (all time points) and 'vitality' (all time points) with $p$ values $<0.041$.

Conclusions This study demonstrates the high burden and a low QoL on the 'the Mental Health Component' domains (or MHC) of caregivers during inpatient rehabilitation. During the early home phase, we found a significant improvement in burden and MHC. Clinicians working with both SCI-patients and caregivers should be aware of the possible high burden and low QoL of caregivers during rehabilitation.
\end{abstract}

Electronic supplementary material The online version of this article (https://doi.org/10.1038/s41393-018-0098-7) contains supplementary material, which is available to authorized users.

Annemiek Petronella Maria Backx

a.p.m.backx@gmail.com

1 Adelante Rehabilitation Centre, Hoensbroek, The Netherlands

2 REVAL-Rehabilitation Research Center-BIOMED, Biomedical Research Institute, Faculty of Medicine and Life Sciences, Hasselt University, Hasselt, Belgium

3 Department of Healthcare, PXL University College Hasselt, Hasselt, Belgium

4 St. Maartenskliniek, Nijmegen, The Netherlands

5 Adelante Centre of Expertise in Rehabilitation and Audiology, Hoensbroek, The Netherlands

6 Department of Rehabilitation Medicine, Maastricht University, Research School CAPHRI, Maastricht, The Netherlands

\section{Introduction}

Patients with a Spinal Cord Injury (SCI) will encounter sudden and severe motor, sensory and/or autonomic dysfunction. Therefore, SCI usually leads to a long-lasting, serious disability, which requires rehabilitation. After rehabilitation, many patients with SCI continue to depend on physical, psychological and/or social support [1-4]. Since the availability of formal care (i.e., professional care provided by a formal service system) is decreasing, the well-being of patients with SCI depends upon care from informal caregivers (the persons caring for the patient outside of a formal service system) [5]. However, informal caregivers often experience a high level of burden due to the demands associated with care of chronically disabled patients, in addition to the stressors in their own life. Caregivers faced with a high level of burden can experience 
a decline in their own physical and/or psychological health [6-10]. This may also have a major impact on their social well-being [7, 9].

The central factors known to increase burden on an informal caregiver of a patient with SCI are: large amounts of support needed for Activities of Daily Living (ADL) of the patient, poorer psychosocial status of the patient with SCI, more recent occurrence of the SCI, the patient receiving professional support, and a greater age and female gender of the caregiver [2]. It has been reported that caregiving spouses experience high levels of physical and emotional stress, burn-out, fatigue, anger, resentment and depression, and lower well-being, as compared to noncaregiving spouses [10]. Cross-sectional data on quality of life (QoL) of caregivers of patients with SCI show some specific domains of QoL to be more affected than others. In particular, caregivers reported low scores on the domains of 'bodily pain', 'vitality', 'emotional role functioning', 'physical role functioning' and 'mental health' but not 'social functioning' [7, 9]. It is also known, from another patient group (palliative cancer), that an informal caregivers' mental health can be related to their satisfaction with formal care of the patient [11].

Most available data on QoL and burden of caregivers of patients with SCI has been collected in cross-sectional studies of varying quality. However, to learn more about the course of QoL and burden, a longitudinal study is required. The first longitudinal study on the course of QoL and burden of caregivers of patients with a SCI in the first years after injury was performed on Australian SCI caregivers. The study had a relatively small patient group and high attrition rate. They reported a low score on the mental component summary score of the SF-36, which improved significantly 6 weeks post rehabilitation discharge. Caregiver burden was found to be high and showed no significant change over the study period [12]. However, due to differences in rehabilitation care, professional support in the subacute/chronic phase, and cultural differences between countries, these results can not necessarily be generalized to caregivers all over the world.

With regard to Dutch caregivers of patients with SCI, little is known about their burden, QoL and satisfaction with care directly post injury and about the course of these issues during and after rehabilitation. Therefore, the main objective of the present prospective, longitudinal cohort study is to evaluate the course of the QoL, the burden and the satisfaction with care of caregivers of patients with SCI during rehabilitation and up to 1.5 years after rehabilitation. In the present study, we focus on the informal caregivers of patients with a SCI, who may be at an even greater risk of negative effects than other informal caregivers because of the unique challenges and the long period of caregiving associated with the care of patients with SCI [8]. We hypothesized that QoL of the caregivers is lower than QoL in the general Dutch population at the start of rehabilitation. Moreover, we hypothesized that QoL and satisfaction with care would increase in the 1.5 years after rehabilitation. The last hypothesis is that QoL or low care satisfaction would have a negative correlation with burden.

\section{Methods}

\section{Participants}

All caregivers of patients with SCI who were admitted to the SCI rehabilitation unit of Adelante Rehabilitation Centre between January 2013 and April 2014 were invited to participate in this study. Adelante Rehabilitation Centre is one of eight specialized rehabilitation centers in the Netherlands providing care to SCI of all subgroups (i.e., paraplegic and tetraplegic, complete and incomplete, and traumatic and non-traumatic). Inclusion criteria were: caregivers who are aged 18 years and older, taking care of a patient with a newly acquired SCI, and are able to understand the Dutch or English language. In case of multiple caregivers for one patient, the patient identified the caregiver who was mainly responsible and most intensively involved. In the case that the included caregiver was no longer the primary caregiver, this caregiver was excluded from the study and considered lost to follow-up. Caregivers were also excluded in case patients with SCI had a life expectancy of $<12$ months. All caregivers received standard care mainly comprising counseling by a social worker, and a group information/education program during the rehabilitation treatment of the patient with SCI. In the follow-up period the caregivers received no rehabilitation care.

Sample size was based on recruiting all subjects available during the inclusion period. Sample size could not be calculated a priori, due to the lack of information about variance and correlation patterns among the repeated measurements [13].

The study was approved by the medical ethical committee of Maastricht University Medical Centre/Maastricht University and Adelante Rehabilitation Centre (METC 124-108; MEC-09-12). Informed consent was obtained from both the caregiver and the patient with SCI before participation. We certify that all applicable institutional and governmental regulations concerning the ethical use of human volunteers were followed during the course of this research. 


\section{Design and procedure}

The data were collected at five time points, including start of inpatient rehabilitation of the patient with SCI (T1), discharge of the patient with SCI from rehabilitation (T2), 8 weeks after discharge (T3), 6 months after discharge (T4) and 18 months after discharge (T5). The data were collected through questionnaires concerning QoL, burden, and satisfaction with care. The questionnaires were sent by mail with a return envelope. In case of no response, the participant was contacted by telephone after 14 days and asked to respond.

\section{Outcome measures}

\section{6-Item Short-form Health Survey (SF-36)}

QoL of the caregiver of a SCI patient was measured using the 36-Item Short-form health Survey (SF-36) [14]. The Dutch translation of this questionnaire is validated, reliable,

Fig. 1 Participant flowdiagram

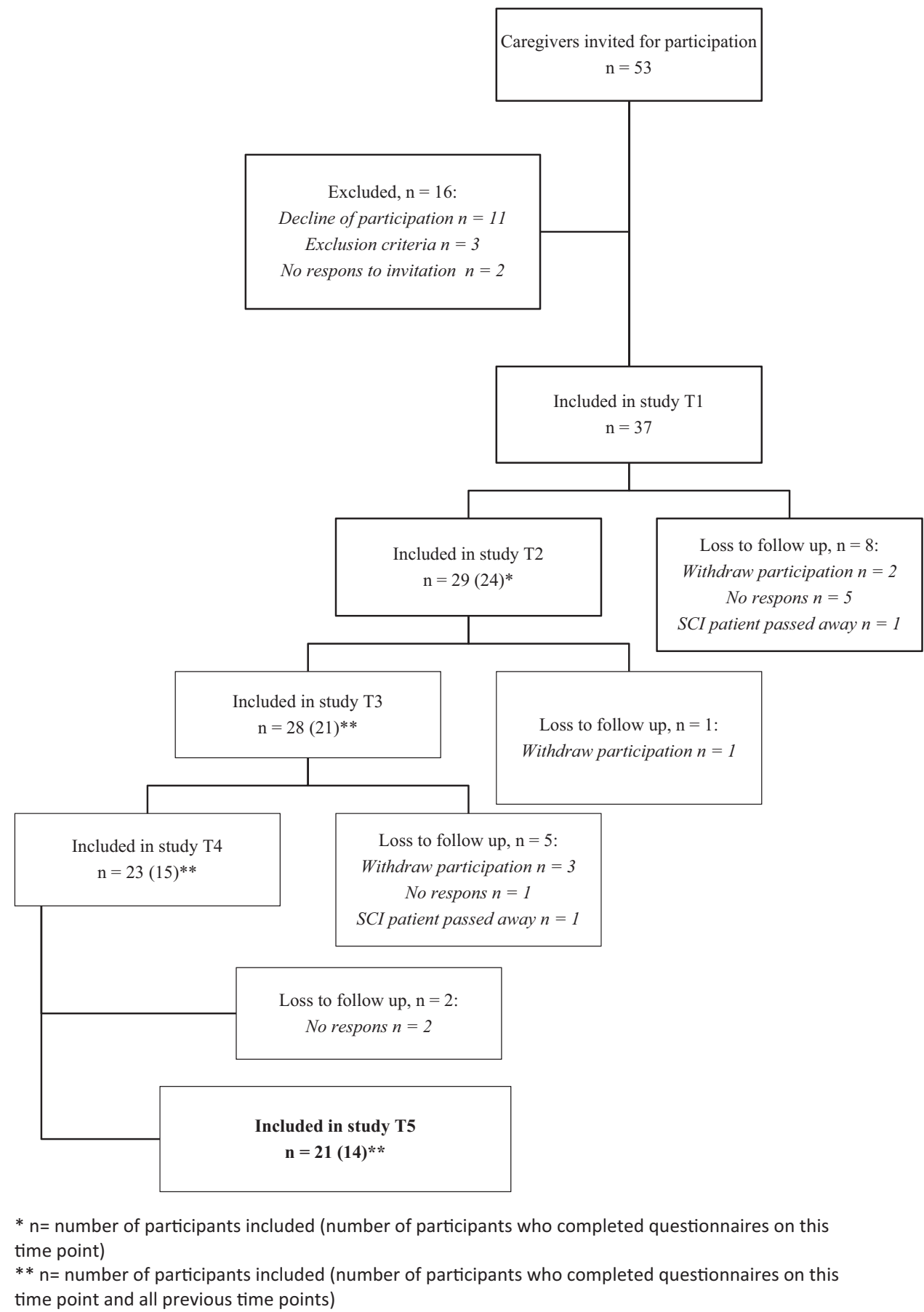


widely used and can be self-administered. The SF-36 assesses 8 domains of Health Related Quality of Life (HRQoL): physical functioning, physical role functioning, bodily pain, health perceptions, vitality, social functioning, emotional role functioning and mental health. The maximum score of 100 corresponds to a perfect HRQoL. The internal reliability is found to be moderate to high $[14,15]$.

\section{Caregiver strain index}

Caregiver burden was measured using the Caregiver Strain Index (CSI) [16]. This questionnaire is short and commonly used to measure caregiver burden [17]. The CSI showed good reproducibility and moderate responsiveness in Dutch stroke caregivers. Scores range from 0 to 13 points; a score of $>6$ is indicative for a high level of burden [16-18].

\section{Caregivers' satisfaction with stroke care questionnaire}

Satisfaction with care of the caregiver was measured using the Caregivers' Satisfaction with Stroke Care Questionnaire (C-SASC) [19]. The C-SASC consists of 19 items. Satisfaction with inpatient care was measured by the hospital subscale (8 items), which consists of questions such as "I have been treated with kindness and respect by the staff" and "I received all the information I wanted". After discharge, satisfaction with (rehabilitation) care was measured using the home subscale (11 items). This subscale evaluates the care regarding/after discharge and outpatient care delivered by the rehabilitation professionals. The home subscale consists of questions such as "Things were well prepared for when the patient for whom I care returns home" [19]. For the C-SASC hospital scale of Dutch caregivers of stroke patients Cronbach's $\alpha$ was found to be high, and convergent and divergent validity tests indicated strong construct validity [19]. For the home scale, no data on validity are available. Although the Caregiver's Satisfaction with Stroke Care Questionnaire was developed for caregivers of persons with stroke, it consists of non-strokespecific questions. Because of these non-disease-related questions, this questionnaire was considered to be most suitable for this study to examine the satisfaction with care of the caregiver. Satisfaction was not measured at T1 due to low amount of rehabilitation care at this time point. The hospital scale was taken at T2 (discharge). The home scale was used to evaluate the satisfaction with care after discharge of the patient (T3, T4, T5). Because the C-SASC questionnaire is an ordinal scale, we chose to produce median scores of the caregivers using this scale.

In addition to the questionnaires, patient characteristics (i.e., lesion level, lesion completeness and functional status
(SCIM: Spinal Cord Independence Measure [20]), as well as caregiver characteristics (i.e., age, gender, living together/relation with the patient with SCI, education, employment and health) were collected as they were considered important to describe the participants at baseline [2].

\section{Data analysis}

Descriptive statistics were calculated for the outcome measures, and the caregiver-related and patient-related characteristics. Non-parametrical tests were performed due to non-normally distributed data, the relatively low number of participants and ordinal data for the CSI and C-SASC. Missingness of the data were analyzed using Little's test for Missing Completely at Random (MCAR). Item imputation was used in case of random missing values according to questionnaire specific instructions. One-sample Wilcoxon signed-rank test was performed to test significant differences between the QoL of the participants of this study and (the known constant of) the QoL of the general Dutch population. Friedman tests were conducted on the caregiver scores of all 8 domains of the SF-36 at all time points, caregiver scores on the CSI at all time points, and caregiver scores on the C-SASC at T2-T5 to examine the course of burden, QoL and satisfaction with care. The level of significance was set at alpha $<0.05$ for the One-sample Wilcoxon signed-rank test and the Friedman test. Post hoc pairwise comparisons were performed with Bonferroni-adjusted $p$ values. To select which correlations would be tested, we first plotted all domains of the SF-36, and the C-SASC with the CSI in scatterplots. Suggested correlations between caregiver burden and domains of QoL or satisfaction with care were tested using Bonferroni adjusted $p$-values on Spearman's rho, with an $\mathrm{r}>0.60$ being considered as a strong correlation.

All the data were analyzed using IBM SPSS software version 23 .

\section{Results}

\section{Data}

The data will be made available upon request by the first author.

\section{Participants}

Out of the 53 caregivers invited, 37 were included in this study. Eleven caregivers refused to participate, mostly because they were too busy and had concerns about the 
Table 1a Baseline characteristics of the caregivers of patients with SCI for all participants $(n=37)$ and for the participants who completed the questionnaires on all time points

\begin{tabular}{|c|c|c|}
\hline Characteristic & $\begin{array}{l}\text { Participants } \\
n=37\end{array}$ & $\begin{array}{l}\text { Participants }^{\mathrm{a}} \\
n=14\end{array}$ \\
\hline Age caregiver in years (mean, sd) & $57(12)$ & $60(10)$ \\
\hline Male, $n(\%)$ & $7(18)$ & $1(7)$ \\
\hline $\begin{array}{l}\text { Caregiver living together with patient, } \\
n(\%)\end{array}$ & $27(71)$ & $10(71)$ \\
\hline \multicolumn{3}{|l|}{ Relationship to SCI patient } \\
\hline Married with patient, $n(\%)$ & $21(55)$ & $9(64)$ \\
\hline Unmarried partner of patient, $n,(\%)$ & $3(8)$ & $0(0)$ \\
\hline Sibling, $n(\%)$ & $4(11)$ & $0(0)$ \\
\hline Parent of patient, $n(\%)$ & $4(11)$ & $3(21)$ \\
\hline Child of patient, $n(\%)$ & $5(13)$ & $2(14)$ \\
\hline Good health, $n(\%)$ & $30(79)$ & $13(93)$ \\
\hline Employed, $n(\%)$ & $19(53)$ & $6(43)$ \\
\hline $1-12 \mathrm{~h}^{\mathrm{b}}$ paid employment, $n(\%)$ & $2(5)$ & $1(7)$ \\
\hline $13-23 \mathrm{~h}^{\mathrm{b}}$ paid employment, $n(\%)$ & $8(21)$ & $2(14)$ \\
\hline $24-35 \mathrm{~h}^{\mathrm{b}}$ paid employment, $n(\%)$ & $7(18)$ & $3(21)$ \\
\hline$<35 \mathrm{~h}^{\mathrm{b}}$ paid employment, $n(\%)$ & $2(5)$ & $0(0)$ \\
\hline \multicolumn{3}{|l|}{ Education level (highest completed), $n(\%)$} \\
\hline Low, $n(\%)$ & $9(24)$ & $3(21)$ \\
\hline Intermediate /vocational, $n(\%)$ & $18(49)$ & $9(64)$ \\
\hline High, $n(\%)$ & $10(27)$ & $2(14)$ \\
\hline $\begin{array}{l}\text { Caregiver for patient before SCI, } \\
n(\%)\end{array}$ & $22(60)$ & $6(43)$ \\
\hline
\end{tabular}

extra burden. Two caregivers did not respond to the invitation to participate. Three patients with SCI did not have a caregiver, so they did not meet the inclusion criteria (see Fig. 1 for participant flow). The caregiver and patient characteristics at baseline are shown in Table 1a, b. Mean duration of rehabilitation treatment (inpatient + outpatient) was $136 \pm 89$ days. During the study, 16 participants (43\%) were lost to follow-up; in 6 cases this was because of withdrawal, 8 participants stopped responding and 2 patients died. This may have led to an attrition bias. A total of 124 questionnaires were returned, 17 questionnaires were not totally completed (14\%). The data were missing at random (Little's test for MCAR, $P<0.05$ ). Item imputation was performed for SF-36 and C-SASC according to questionnaire specific instructions. Because of the small sample, and high drop-out rate, the study results have low power and may be biased. Only the participants who completed questionnaires on all time points $(n=14)$ were included in the statistical analysis. Mean follow-up duration was $22 \pm 3$ months
Table 1b Baseline characteristics of the patients with SCI for all patients $(n=37)$ and for the patients for whom the questionnaires were completed by the caregiver on all time points

\begin{tabular}{lll}
\hline Characteristic & Patients $n=37$ & Patients $^{\mathrm{a}} n=14$ \\
\hline Age in years (mean, sd) & $58(16)$ & $60(20)$ \\
Male, $n(\%)$ & $27(73)$ & $11(79)$ \\
Tetraplegia, $n(\%)$ & $11(29)$ & $2(14)$ \\
ASIA classification, $n(\%)$ & & \\
AIS A, n (\%) & $12(312)$ & $5(36)$ \\
AIS B, n (\%) & $1(3)$ & $1(7)$ \\
AIS C, n (\%) & $7(18)$ & $2(14)$ \\
AIS D, n (\%) & $17(45)$ & $6(43)$ \\
SCIM level at T1, mean (sd) & $28(25)$ & $55(23)$ \\
\hline
\end{tabular}

SCIM spinal cord independence measure, AIS classification American Spinal Injury Association Impairment Scale, $S D$ standard deviation

${ }^{\text {a }} n=14$ (patients who completed questionnaires on all time points)

\section{Caregivers' quality of life}

Table 2 shows the course of the SF-36 domains of QoL. At the start of rehabilitation, caregivers scored low on the domains 'social functioning', 'emotional role functioning', 'mental health' and 'vitality' (together the 'the Mental Health Component domains, or MHC). In Table 3, the scores of the caregivers (at T1) are shown in comparison with the scores of the Dutch general population [15]. The scores of the caregivers on these four domains at $\mathrm{T} 1$ are significantly lower than the Dutch general population $(p>0.05)$. These initial low scores improved significantly during the follow-up period, with the largest improvement in 'social functioning' (improvement of 44 points from $\mathrm{T} 1$ to $\mathrm{T} 5$, for the total group of participants) and 'emotional role functioning' (improvement of 33 points from T1 to T5, for the total group of participants). Post hoc pairwise comparisons with adjusted $p$-values showed no significant differences between the individual time points.

\section{Caregivers' burden}

During rehabilitation 20 caregivers (54\%) experienced high levels of burden with a median CSI score of 7, IQR [5;10]. This number of highly strained caregivers decreased to 11 $(38 \%)$ at $\mathrm{T} 2,10(36 \%)$ at $\mathrm{T} 3$, and $4(17 \%)$ at $\mathrm{T} 4$. At $\mathrm{T} 5$, one additional caregiver with a high level of burden was found. When taking a look at the 14 participants who completed measurements on all time points, the number of highly strained caregivers decreased from $29 \%$ at $\mathrm{T} 1$ to $14 \%$ at $\mathrm{T} 5$. The portion of highly strained caregivers was tested by the 


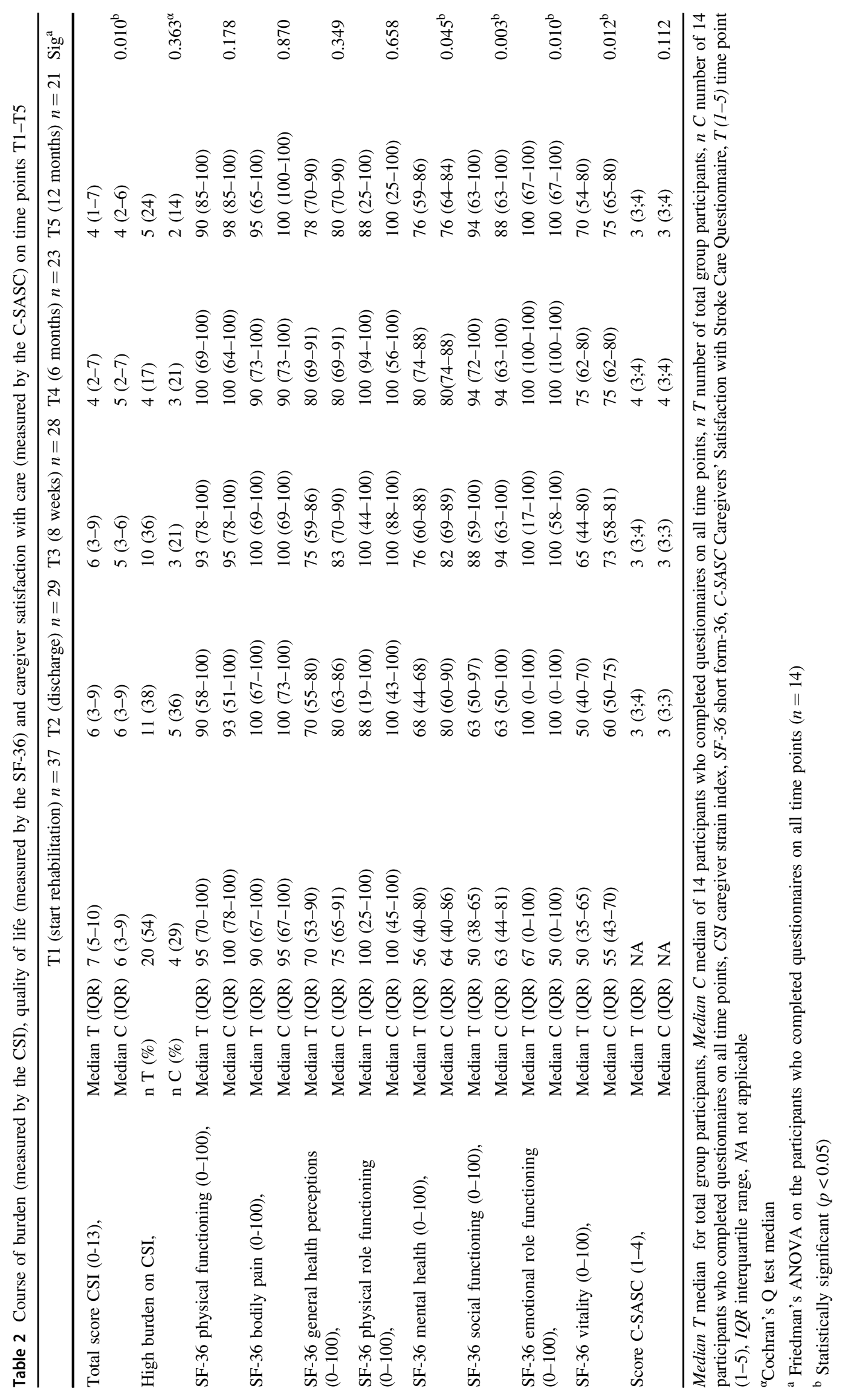


Table 3 Comparison of the caregiver SF-36 scores with the SF-36 scores of the general Dutch population

\begin{tabular}{llll}
\hline SF-36 domain $^{\mathrm{a}}$ & Caregivers $^{\mathrm{b}}$ & ${\text { Netherlands } 1992^{\mathrm{c}}}$ & $P$ value $^{\mathrm{d}}$ \\
\hline Physical functioning, mean (sd) & $85(18)$ & $82(23)$ & $79(26)$ \\
Bodily pain, mean (sd) & $83(21)$ & $73(23)$ & 0.19 \\
General health perceptions, mean (sd) & $69(18)$ & $79(36)$ & 0.21 \\
Physical role functioning, mean (sd) & $69(43)$ & $77(18)$ & 0.12 \\
Mental health, mean (sd) & $57(23)$ & $87(21)$ & 0.35 \\
Social functioning, mean (sd) & $55(27)$ & $84(32)$ & $0.000^{*}$ \\
Emotional role functioning, mean (sd) & $52(45)$ & $67(20)$ & $0.000^{*}$ \\
Vitality, mean (sd) & $49(20)$ & $0.000^{*}$ \\
\hline
\end{tabular}

$S F-36$ short form-36, $T 1$ start of clinical rehabilitation, $s d$ standard deviation

$* p<0.05$

${ }^{\text {a }}$ Range $0-100$ for all domains

${ }^{\mathrm{b}}$ Scores of caregivers at $\mathrm{T} 1$

${ }^{\mathrm{c}}$ Mean scores of Dutch general population [15]

${ }^{\mathrm{d}}$ One-sample Wilcoxon signed-rank test

Cochran's Q test, which did not indicate significant differences among the 5 time points $(p=0.363$; Table 2$)$. The decrease in caregivers' median burden score on the CSI was statistically significant on the Friedman test $(p=0.010$; Table 2). Post hoc pairwise comparisons with adjusted $p$ values showed a significant difference between T2 and T5 (Bonferroni adjusted $p=0.036$ ).

\section{Caregivers' satisfaction with care}

Overall satisfaction with care of caregivers was good (Table 2). Caregivers scored a median of 3 on the C-SASC, IQR [3, 4], which was stable over time.

\section{Correlations of caregiver burden and quality of life/ C-SASC}

Because of the resemblance of the course of CSI and SF-36 domains 'social functioning', 'emotional role functioning', 'mental health' and 'vitality' and their possible correlation statistically significant correlations were tested (Table 4). On the basis of the scatterplots, we did not test the other domains. Moderate to strong negative correlations were found between CSI and these domains of SF-36. We found no correlations between the scores on C-SASC and CSI total scores, except for the T4 measurement.

\section{Discussion}

Informal caregivers are becoming increasingly important for the well-being of patients with a chronic disability, such as those with a SCI. Therefore, attention to the QoL, burden and satisfaction with care of caregivers is a relevant aspect of the total care for patients with a SCI.

The first hypothesis of this study was that "QoL of the caregivers is lower than QoL in the general Dutch population at the start of rehabilitation". The participants in the present study reported a high burden and a low QoL on the MHC during inpatient rehabilitation. The scores of the caregivers on these four domains at $\mathrm{T} 1$ are significantly lower than the Dutch general population $(p<0.05)$.

The hypothesis "that QoL of the caregivers is lower than QoL in the general Dutch population at the start of rehabilitation" is rejected because we found no significant changes in the four other domains of the SF-36. A possible explanation for the low scores on the MHC of the SF-36 might be that the caregivers predominantly provide care of mental origin at this stage, such as giving support, coming to terms with and organizing the new situation, and probably also worrying about the future. The physical care/ support during inpatient rehabilitation is mainly performed by the nursing and paramedical staff.

The second hypothesis was that "QoL and satisfaction with care would increase in the 1.5 years after rehabilitation". During the follow-up, when the patient has been discharged from rehabilitation, MHC and caregiver burden showed a significant improvement. However, we found different patterns for all 8 domains of HRQoL, the hypothesis that "QoL will increase within 1.5 years after rehabilitation" is therefore rejected.

The reason for improvement in the MHC could be related to the improvement of the physical abilities of the patient with SCI and the support and compassion available for the caregiver. Therefore, this early home phase might be characterized by positive feelings regarding future 
Table 4 Correlations between the caregiver burden and the Mental Health Component' domains of the Sf-36, and correlations between the caregiver burden and the caregiver satisfaction with care

\begin{tabular}{llllll}
\hline & $\begin{array}{l}\text { Total score } \\
\text { CSI T1 } n=37\end{array}$ & $\begin{array}{l}\text { Total score } \\
\text { CSI T2 } n=29\end{array}$ & $\begin{array}{l}\text { Total score } \\
\text { CSI T3 } n=28\end{array}$ & $\begin{array}{l}\text { Total score } \\
\text { CSI T4 } n=23\end{array}$ & $\begin{array}{l}\text { Total score } \\
\text { CSI T5 } n=21\end{array}$ \\
\hline SF-36 Mental health & $\mathrm{r}=-0.71^{\mathrm{a}}$ & $\mathrm{r}=-0.64^{\mathrm{a}}$ & $\mathrm{r}=-0.74^{\mathrm{a}}$ & $\mathrm{r}=-0.70^{\mathrm{a}}$ & $\mathrm{r}=-0.67^{\mathrm{a}}$ \\
& $\mathrm{p}=0.000^{\mathrm{b}}$ & $\mathrm{p}=0.001^{\mathrm{b}}$ & $\mathrm{p}=0.000^{\mathrm{b}}$ & $\mathrm{p}=0.002$ & $\mathrm{p}=0.002$ \\
SF-36 Social & $\mathrm{r}=-0.48$ & $\mathrm{r}=-0.57$ & $\mathrm{r}=-0.65^{\mathrm{a}}$ & $\mathrm{r}=-0.70^{\mathrm{a}}$ & $\mathrm{r}=-0.47$ \\
functioning & $\mathrm{p}=0.003$ & $\mathrm{p}=0.003$ & $\mathrm{p}=0.000^{\mathrm{b}}$ & $\mathrm{p}=0.002$ & $\mathrm{p}=0.051$ \\
SF-36 Emotional role & $\mathrm{r}=-0.52$ & $\mathrm{r}=-0.61^{\mathrm{a}}$ & $\mathrm{r}=-0.61^{\mathrm{a}}$ & $\mathrm{r}=-0.50$ & $\mathrm{r}=-0.68^{\mathrm{a}}$ \\
functioning & $\mathrm{p}=0.001^{\mathrm{b}}$ & $\mathrm{p}=0.002$ & $\mathrm{p}=0.001^{\mathrm{b}}$ & $\mathrm{p}=0.041$ & $\mathrm{r}=0.004$ \\
SF-36 Vitality & $\mathrm{r}=-0.54$ & $\mathrm{r}=-0.64^{\mathrm{a}}$ & $\mathrm{r}=-0.67^{\mathrm{a}}$ & $\mathrm{r}=-0.55$ & $\mathrm{p}=0.021$ \\
Score C-SASC & $\mathrm{p}=0.003$ & $\mathrm{p}=0.001^{\mathrm{b}}$ & $\mathrm{p}=0.000^{\mathrm{b}}$ & $\mathrm{p}=-0.71^{\mathrm{a}}$ & $\mathrm{r}=-0.37$ \\
& $\mathrm{NA}$ & $\mathrm{r}=-0.13$ & $\mathrm{r}=-0.29$ & $\mathrm{r}=0.002$ & $\mathrm{p}=0.117$ \\
\hline
\end{tabular}

$T 1$ start of clinical rehabilitation, $T 2$ patient's discharge from rehabilitation, $T 38$ weeks after discharge, $T 46$ months after discharge, $T 518$ months after discharge, $s d$ standard deviation; IQR interquartile range; NA not applicable, CSI Caregiver Strain index, SF-36 Short Form-36, C-SASC Caregivers' Satisfaction with Stroke Care Questionnaire, $M H C$ Mental Health Component' domains

${ }^{a}$ Strong $(r>0.60)$ correlation regarding Spearman's rho

${ }^{\mathrm{b}}$ Statistically significant (Bonferroni adjusted $p<0.002$ )

outcomes. Also, family reunification could increase the mental aspects associated with QoL for the caregivers. This improvement in MHC after rehabilitation is consistent with earlier results of Middleton et al., who showed Australian caregivers of patients with SCI also improved on MHC domain' scores of quality of life during follow-up after rehabilitation [12].

It was also observed that QoL in all domains stabilized after the early home phase. This course is in line with the course of mental health of patients with a SCI, described by Van Leeuwen et al. [21]. They found that mental health increased between the start of active rehabilitation and 3 months later. They also found that the mental health remained stable between 3 months after start rehabilitation and 2 years after discharge [21]. The resemblance of the course of the mental health of the patient and the MHC of the QoL of the caregiver suggests a correlation. A poorer psychosocial status of the patient with SCI is already known to increase burden on an informal caregiver [2]. A high caregiver burden and decreased QoL has been found in other diagnoses, like chronic heart failure [22], traumatic brain injury/polytrauma $[23]^{3}$ and advanced cancer $[11,24]$. In palliative care, patients and their caregivers are sometimes considered a "unit of care" [24], which also seems applicable to the patient with SCI and their caregiver. Especially because the course of the mental health of the patient with SCI and the MHC of the QoL caregiver show the above mentioned similarities.

The last hypothesis was that "QoL or low care satisfaction will have a negative correlation with burden". Moderate to strong negative correlations were found between CSI and the MHC domains of SF-36. We found no correlations between the scores on C-SASC and CSI total scores, except for the T4 measurement. This hypothesis therefore is rejected.

We urge clinicians working in the field of SCI rehabilitation to be aware of the possible high burden and low QoL of caregivers during the rehabilitation phase. It is therefore very important to monitor and regularly check the wellbeing of, not only the patient with SCI, but also the informal caregivers and to recognize high burden, especially during the rehabilitation phase. We advise to inform the caregiver about the high burden and to make sure they know where to find help or support when needed.

To our knowledge, the present study is the first to report on satisfaction of caregivers of patients with SCI with rehabilitation care. This is one of the reasons why a customary tool to examine satisfaction with care of SCI caregivers was non-existent. To measure satisfaction of SCI caregivers with rehabilitation care the Caregivers' Satisfaction with Stroke Care Questionnaire was used [19]. Although this questionnaire was developed for caregivers of stroke patients, it was considered to be adequate for this study. Internal consistency of the scores of the SCI caregivers on the different items of the C-SASC was high for all time points. The overall satisfaction with care of caregivers was good and did not show a consistent correlation with caregiver burden during follow-up.

An important methodological aspect of this study is the way of recruiting the participants. We systematically asked all caregivers of the patients admitted to rehabilitation to participate, instead of waiting for caregivers to respond to a call to participate. Because of this method, the risk of overrepresenting overburdened caregivers is small. 


\section{Study limitations}

Because we aimed to assess the course of burden, QoL and satisfaction with care without emphasizing an intervention, a longitudinal cohort study was the most suitable and best possible design. A shortcoming of this approach is its uncontrolled nature, which may have introduced a selection bias. In addition, the study population is relatively small, and we lost a considerable number of participants during follow-up (43\%). Therefore, the data of this study should be interpreted with caution. Despite these limitations, this study provides initial data worthy of further exploration and stressing the importance of taking into account the QoL and burden of caregivers in rehabilitation. Future research in this field should focus on studyingQoL, burden and satisfaction with care in a larger cohort with a longer follow-up. In this way, the effect of giving care for the longer term on QoL can be studied in more detail.

\section{Conclusion}

This study revealed a high burden and a low QoL regarding the 'Mental Health Component domains' of caregivers of patients with SCI during inpatient rehabilitation. During the early home phase, we found a significant improvement of burden and 'mental' quality of life. Clinicians working in the field of SCI rehabilitation should be aware of the possible high burden and low QoL of caregivers during the rehabilitation phase We advise rehabilitation professionals to inform the caregivers well about the high burden and to make sure they know where to find help or support when needed.

Authors contributions $\mathrm{AB}$ was responsible for designing the protocol, writing the protocol, including patients, analyzing the data, interpreting the results, writing article, submitting article. AS was responsible for designing the protocol, contributed to interpreting the results and provided feedback on the article. HB contributed to designing the protocol, including patients, interpreting results and provided feedback on the article. HB contributed to including patients, analyzing the data, interpreting the results and provided feedback on the article.

\section{Compliance with ethical standards}

Conflict of interest The authors declare that they have no conflict of interest.

\section{References}

1. Biering-Sorensen T, Hansen RB, Biering-Sorensen F. Home aids and personal assistance 10-45 years after spinal cord injury. Spinal Cord. 2009;47:405-12.

2. Post MW, Bloemen J, de Witte LP. Burden of support for partners of persons with spinal cord injuries. Spinal Cord. 2005;43:311-9.
3. Smith EM, Boucher N, Miller WC. Caregiving services in spinal cord injury: a systematic review of the literature. Spinal Cord. 2016;54:562-9.

4. van Loo MA, Post MW, Bloemen JH, van Asbeck FW. Care needs of persons with long-term spinal cord injury living at home in the Netherlands. Spinal Cord. 2010;48:423-8.

5. Van Durme T, Macq J, Jeanmart C, Gobert M. Tools for measuring the impact of informal caregiving of the elderly: a literature review. Int J Nurs Stud. 2012;49:490-504.

6. Adelman RD, Tmanova LL, Delgado D, Dion S, Lachs MS. Caregiver burden: a clinical review. JAMA. 2014;311:1052-60.

7. Blanes L, Carmagnani MI, Ferreira LM. Health-related quality of life of primary caregivers of persons with paraplegia. Spinal Cord. 2007;45:399-403.

8. Schulz R, Czaja SJ, Lustig A, Zdaniuk B, Martire LM, Perdomo D. Improving the quality of life of caregivers of persons with spinal cord injury: a randomized controlled trial. Rehabil Psychol. 2009;54:1-15.

9. Unalan H, Gencosmanoglu B, Akgun K, Karamehmetoglu S, Tuna $\mathrm{H}$, Ones K, et al. Quality of life of primary caregivers of spinal cord injury survivors living in the community: controlled study with short form-36 questionnaire. Spinal Cord. 2001;39:318-22.

10. Weitzenkamp DA, Gerhart KA, Charlifue SW, Whiteneck GG, Savic G. Spouses of spinal cord injury survivors: the added impact of caregiving. Arch Phys Med Rehabil. 1997;78:822-7.

11. Morishita M, Kamibeppu K. Quality of life and satisfaction with care among family caregivers of patients with recurrent or metastasized digestive cancer requiring palliative care. Support Care Cancer. 2014;22:2687-96.

12. Middleton JW, Simpson GK, De Wolf A, Quirk R, Descallar J, Cameron ID. Psychological distress, quality of life, and burden in caregivers during community reintegration after spinal cord injury. Arch Phys Med Rehabil. 2014;95:1312-9.

13. Guo Y, Logan HL, Glueck DH, Muller KE. Selecting a sample size for studies with repeated measures. BMC Med Res Methodol. 2013;13:100.

14. McHorney CA, Ware JE Jr., Lu JF, Sherbourne CD. The MOS 36item Short-Form Health Survey (SF-36): III. Tests of data quality, scaling assumptions, and reliability across diverse patient groups. Med Care. 1994;32:40-66.

15. van der Zee K, Sanderman R, Heyink J. De psychometrische kwaliteiten van de MOS 36-item short form health survey (SF-36) in een Nederlands populatie. Tijdschr voor Soc Gezondh. 1994;4:183-91.

16. Robinson BC. Validation of a caregiver strain index. J Gerontol. 1983;38:344-8.

17. Post MW, Festen H, van de Port IG, Visser-Meily JM. Reproducibility of the Caregiver Strain Index and the Caregiver Reaction Assessment in partners of stroke patients living in the Dutch community. Clin Rehabil. 2007;21:1050-5.

18. Visser-Meily JM, Post MW, Riphagen II, Lindeman E. Measures used to assess burden among caregivers of stroke patients: a review. Clin Rehabil. 2004;18:601-23.

19. Cramm JM, Strating MM, Nieboer AP. Validation of the caregivers' satisfaction with Stroke Care Questionnaire: C-SASC hospital scale. J Neurol. 2011;258:1008-12.

20. Catz A, Itzkovich M, Agranov E, Ring H, Tamir A. SCIM--spinal cord independence measure: a new disability scale for patients with spinal cord lesions. Spinal Cord. 1997;35:850-6.

21. van Leeuwen CM, Hoekstra T, van Koppenhagen CF, de Groot $S$, Post MW. Trajectories and predictors of the course of mental health after spinal cord injury. ArchPhys Med Rehabil. 2012;93:2170-6. 
22. Luttik ML, Jaarsma T, Veeger NJ, van Veldhuisen DJ. For better and for worse: Quality of life impaired in HF patients as well as in their partners. Eur J Cardiovasc Nurs. 2005;4:11-4.

23. Griffin JM, Lee MK, Bangerter LR, Van Houtven $\mathrm{CH}$, Friedemann-Sanchez G, Phelan SM, et al. Burden and mental health among caregivers of veterans with traumatic brain injury/ polytrauma. AmJ Orthopsychiatry. 2017;87:139-48.
24. Ullrich A, Ascherfeld L, Marx G, Bokemeyer C, Bergelt C, Oechsle K. Quality of life, psychological burden, needs, and satisfaction during specialized inpatient palliative care in family caregivers of advanced cancer patients. BMC Palliat Care. 2017;16:31. 